Supporting Information

\title{
Solvatochromic parameters of deep eutectic solvents: effect of different carboxylic acids as hydrogen bond donor
}

Amos K. Dwamena and Douglas E. Raynie*

Department of Chemistry and Biochemistry, South Dakota State University, Brookings, SD 57007, USA

* Corresponding author

Tel.: +16056886361

E-mail address: douglas.raynie@sdstate.edu 


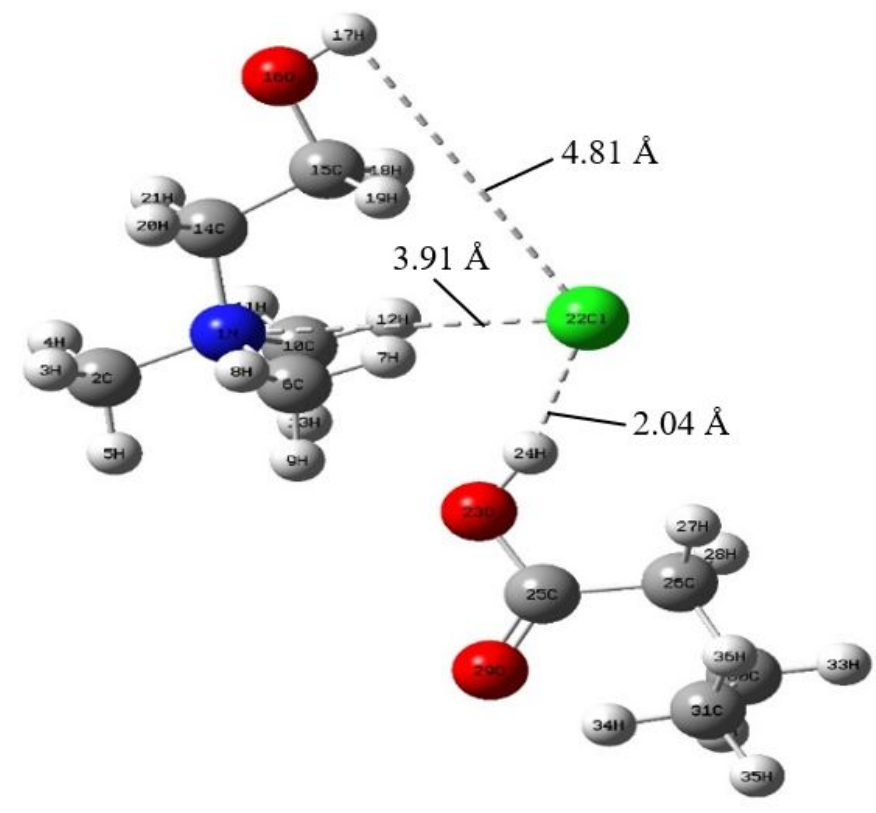

Fig. S1. Optimized equilibrium geometry structure of [Ch]Cl:BA (1:1) DES. Atoms are colored according to type: $\mathrm{C}$ (gray), $\mathrm{O}$ (red), $\mathrm{N}$ (blue), $\mathrm{H}$ (white), and $\mathrm{Cl}$ (light green). Bond angle between C14-C15-O16 (102.69 $\left.{ }^{\circ}\right)$, C15-O16-H17 (107.40 $\left.{ }^{\circ}\right)$, C2-N1-C14 (107.94 ${ }^{\circ}$ ), and N1C14-C15 $\left(116.54^{\circ}\right)$.

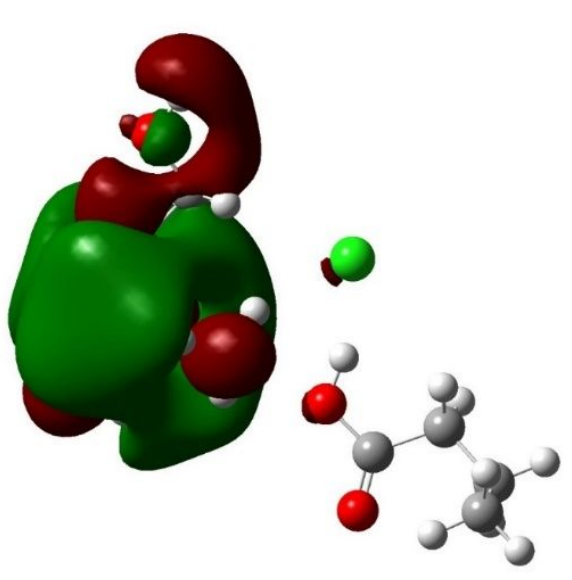

a)

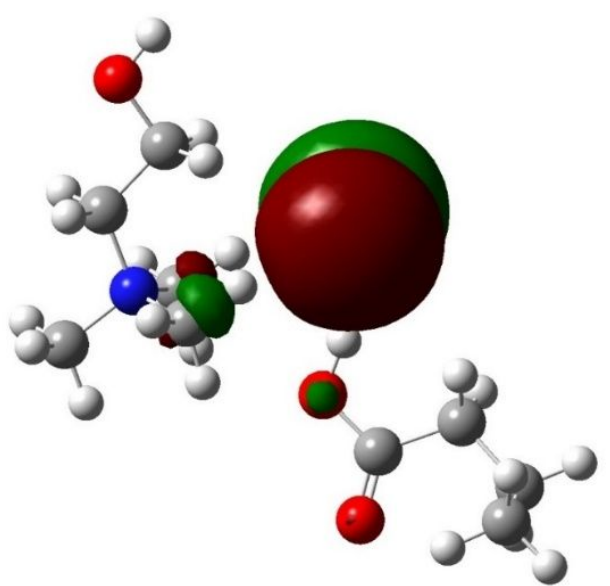

b)

Fig. S2. Frontier molecular orbitals of [Ch]Cl:BA (1:1) DES: (a) HOMO and (b) LUMO 


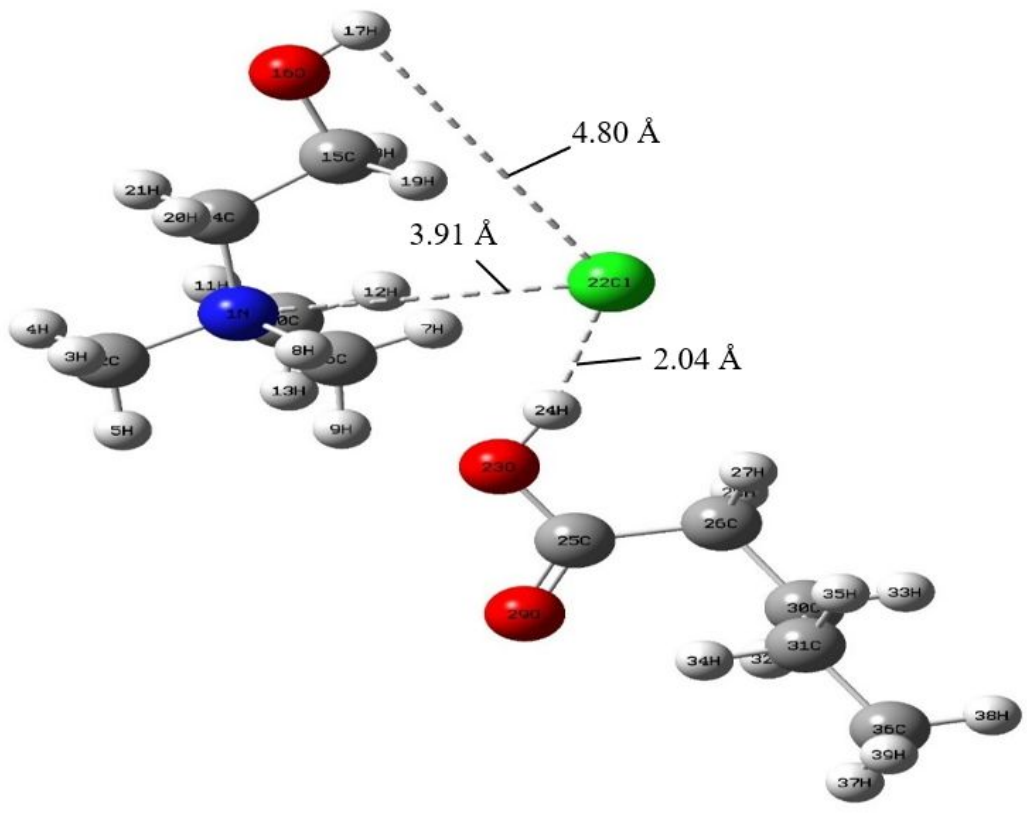

Fig. S3. Optimized equilibrium geometry structure of [Ch]Cl:VA (1:1) DES. Atoms are colored according to type: $\mathrm{C}$ (gray), $\mathrm{O}$ (red), $\mathrm{N}$ (blue), $\mathrm{H}$ (white), and $\mathrm{Cl}$ (light green). Bond angle between C14-C15-O16 (102.69 $\left.{ }^{\circ}\right)$, C15-O16-H17 (107.40 $\left.{ }^{\circ}\right), \mathrm{C} 2-\mathrm{N} 1-\mathrm{C} 14$ (107.96 $\left.{ }^{\circ}\right)$, and N1C14-C15 $\left(116.53^{\circ}\right)$.
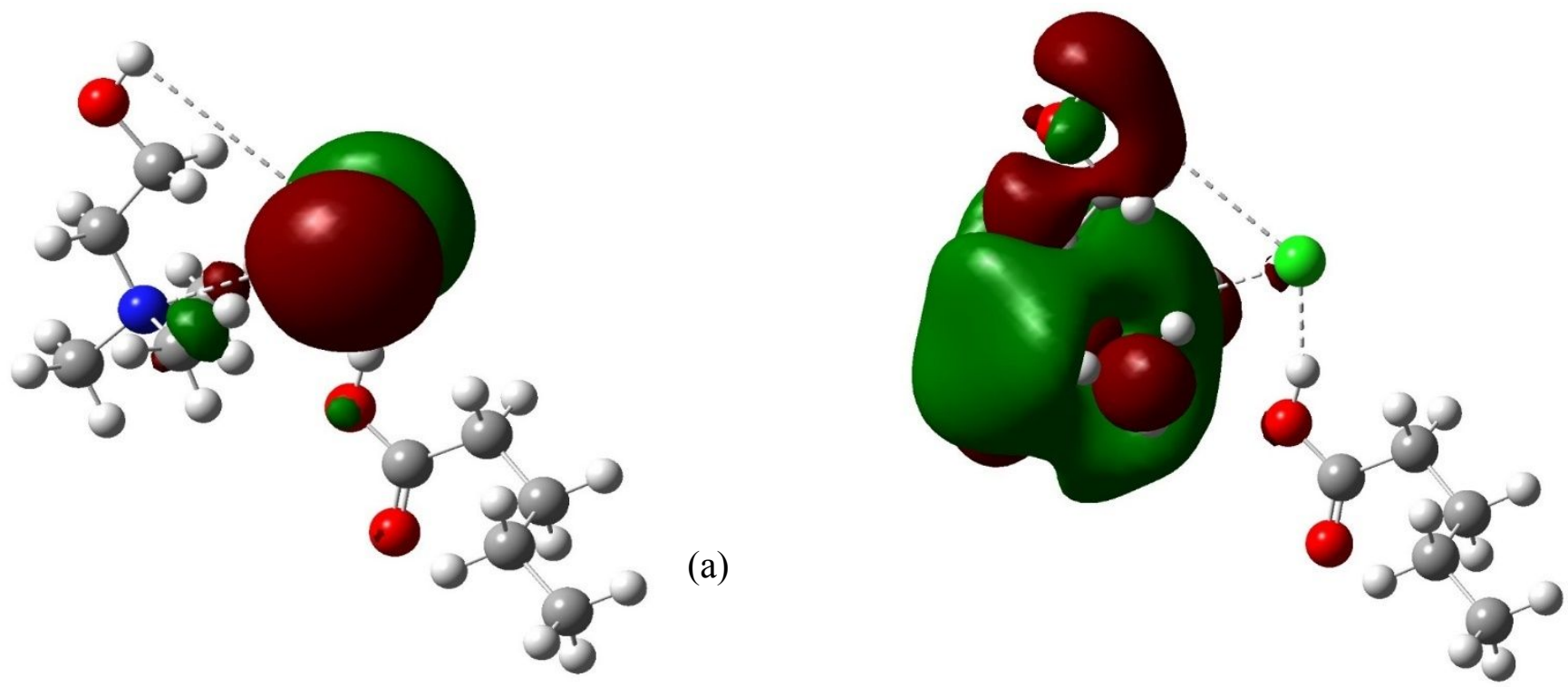

Fig. S4. Frontier molecular orbitals of [Ch]Cl:VA DES: (a) HOMO and (b) LUMO 


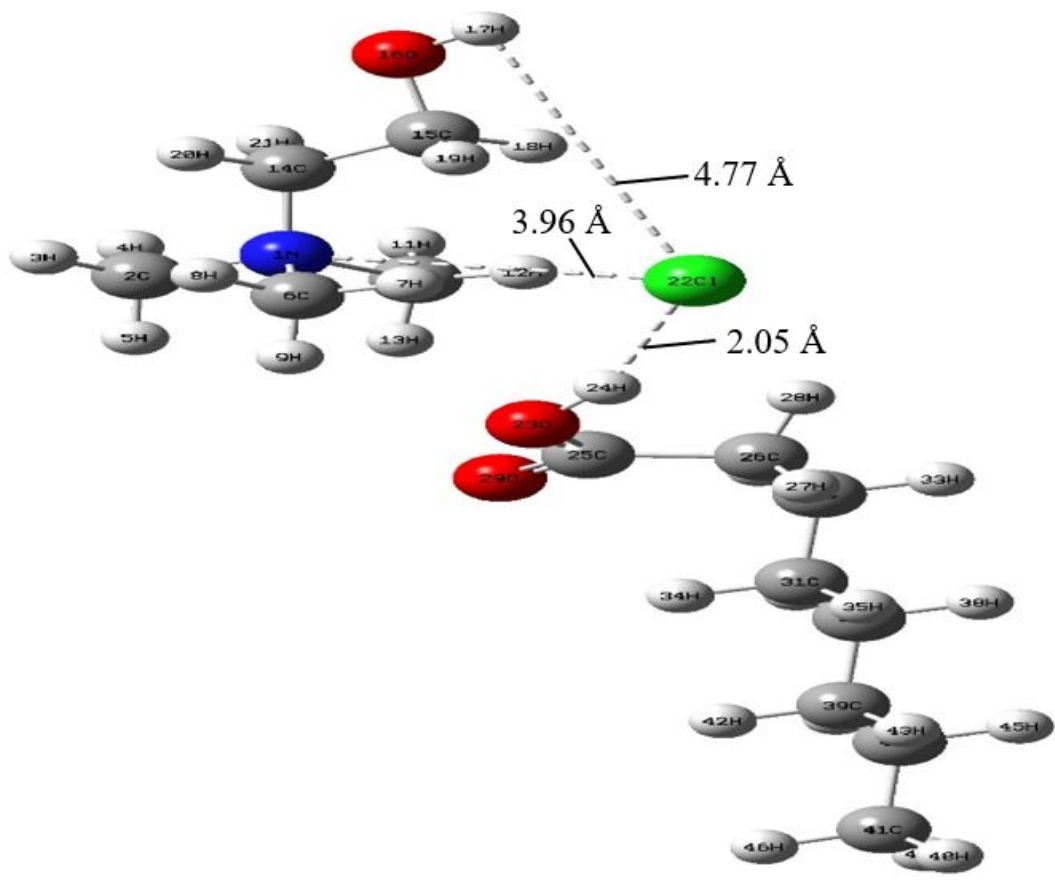

Fig. S5. Optimized equilibrium geometry structure of [Ch]Cl:CA (1:1) DES. Atoms are colored according to type: $\mathrm{C}$ (gray), $\mathrm{O}$ (red), $\mathrm{N}$ (blue), $\mathrm{H}$ (white), and $\mathrm{Cl}$ (light green). Bond angle between C14-C15-O16 (102.77 $\left.{ }^{\circ}\right)$, C15-O16-H17 (107.33 $\left.{ }^{\circ}\right), \mathrm{C} 2-\mathrm{N} 1-\mathrm{C} 14$ (108.00 $\left.{ }^{\circ}\right)$, and N1C14-C15 $\left(116.44^{\circ}\right)$.
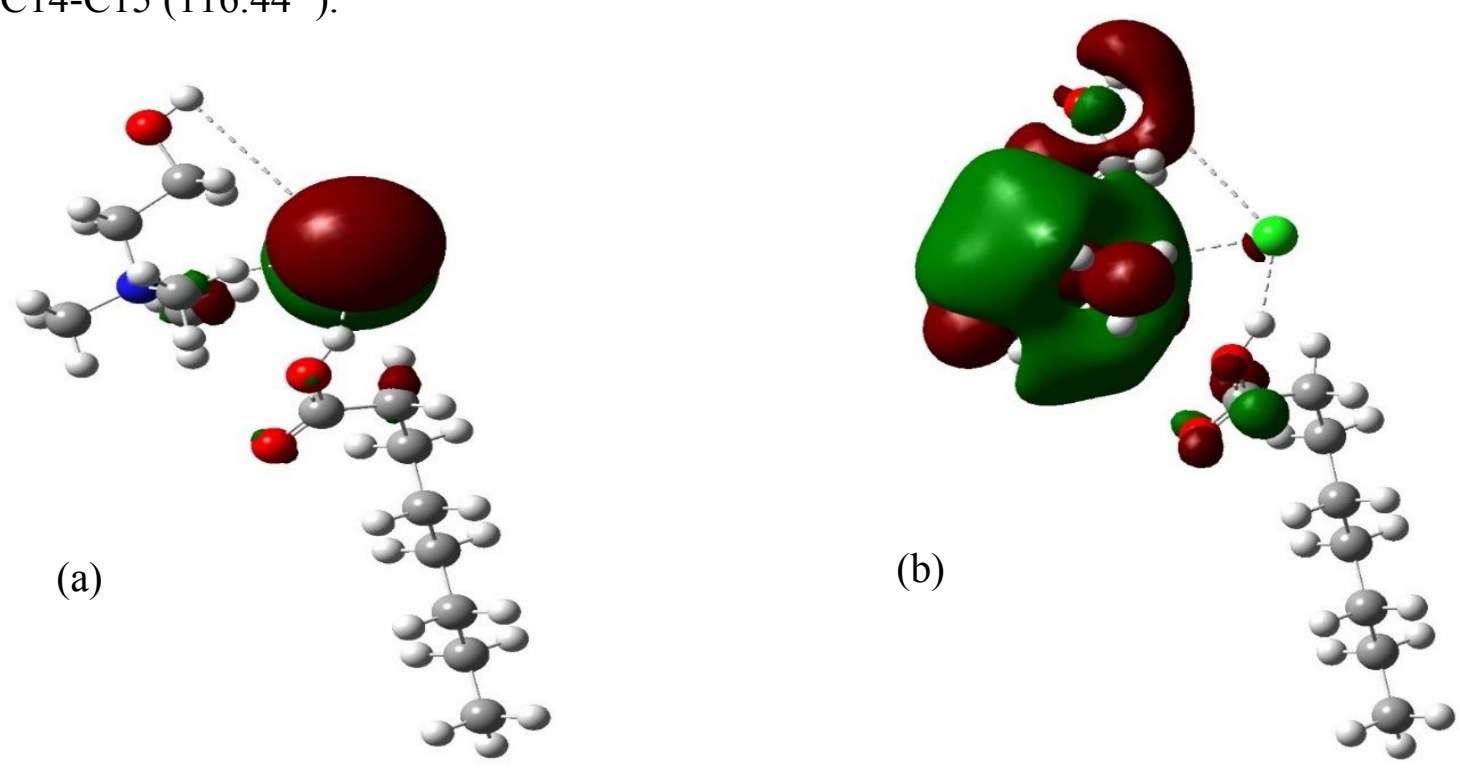

Fig. S6. Frontier molecular orbitals of [Ch]Cl:CA DES: (a) HOMO and (b) LUMO 


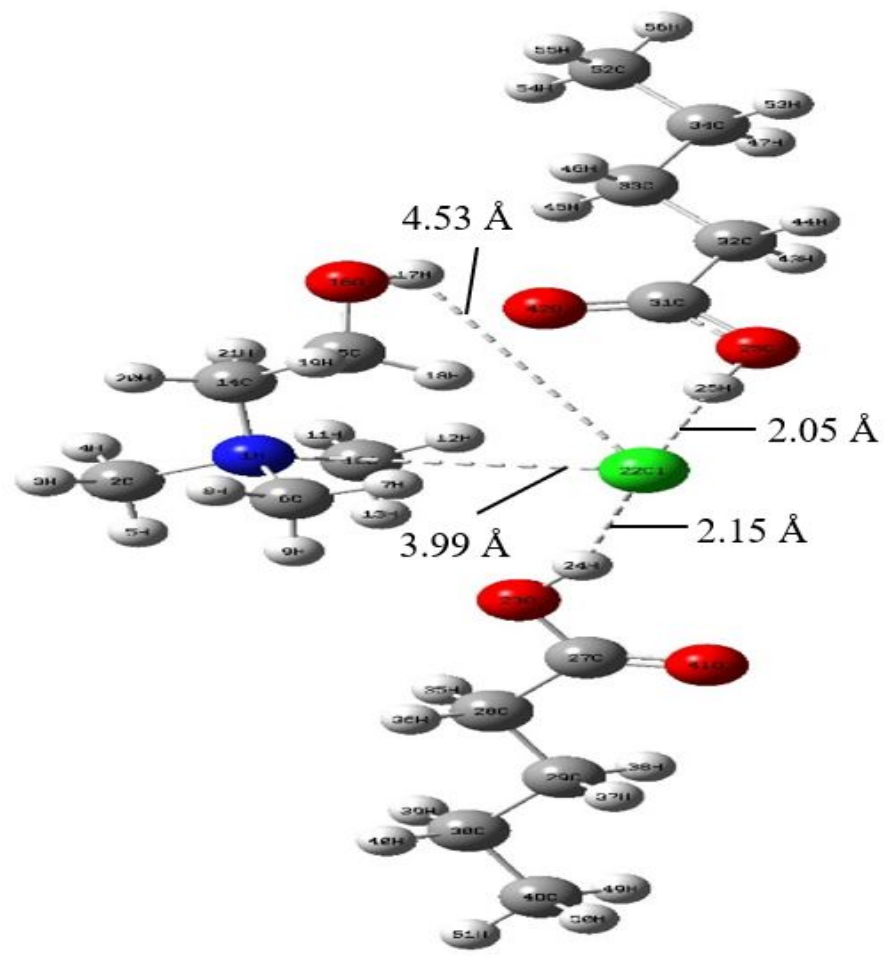

Fig. S7. Optimized equilibrium geometry structure of [Ch]Cl:VA (1:2) DES. Atoms are colored according to type: $\mathrm{C}$ (gray), $\mathrm{O}$ (red), $\mathrm{N}$ (blue), $\mathrm{H}$ (white), and $\mathrm{Cl}$ (light green). Bond angle between C14-C15-O16 (107.99 $\left.{ }^{\circ}\right)$, C15-O16-H17 (105.01 $\left.{ }^{\circ}\right)$, C2-N1-C14 (108.72 ${ }^{\circ}$ ), and N1C14-C15 (116.09 $\left.{ }^{\circ}\right)$.
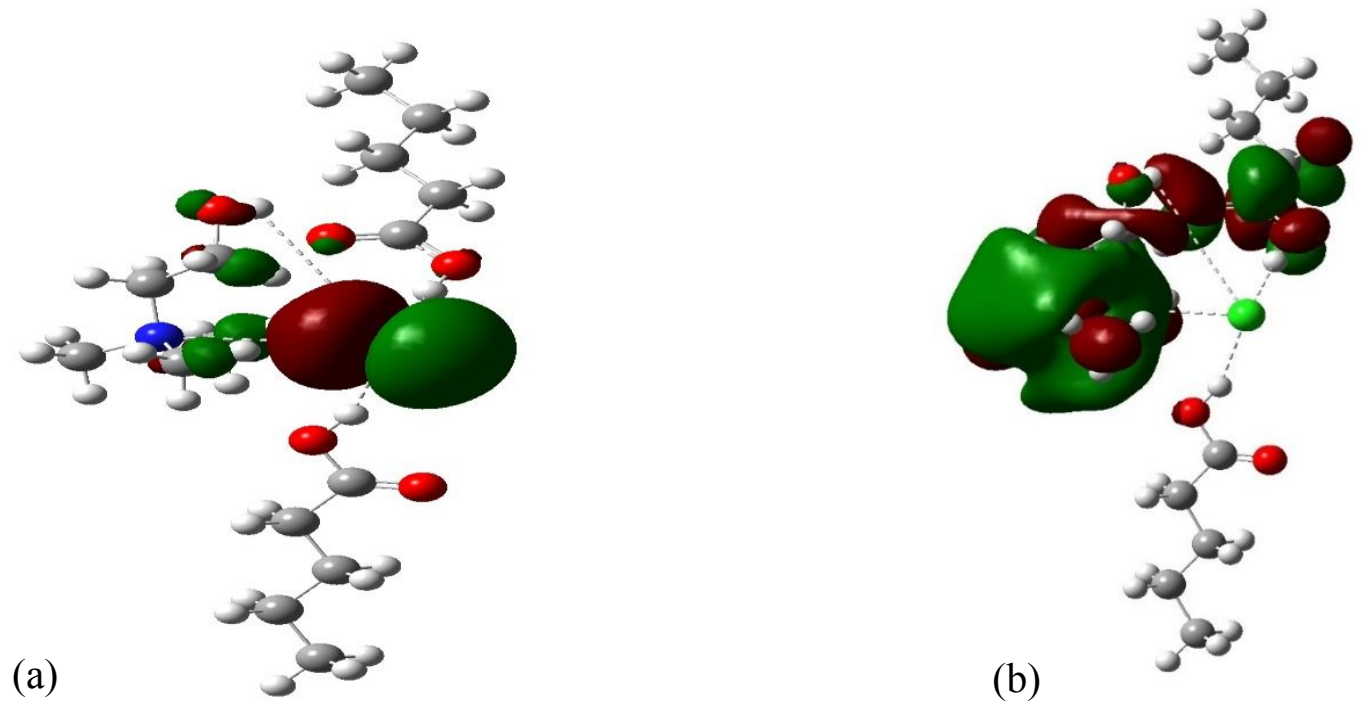

Fig. so. rrontier molecular orbitals of [Ch]Cl:VA (1:2) DES: (a) inviviv and (b) LUMO 


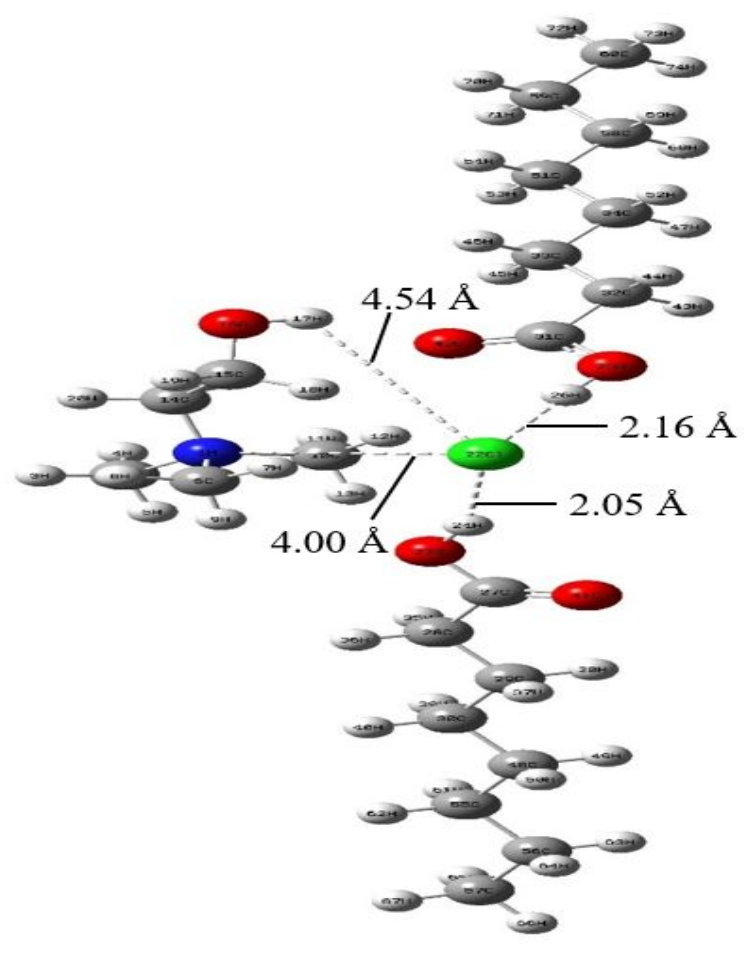

Fig. S9. Optimized equilibrium geometry structure of [Ch]Cl:CA (1:2) DES. Atoms are colored according to type: $\mathrm{C}$ (gray), $\mathrm{O}$ (red), $\mathrm{N}$ (blue), $\mathrm{H}$ (white), and $\mathrm{Cl}$ (light green). Bond angle between C14-C15-O16 (108.05 ${ }^{\circ}$ ), C15-O16-H17 (105.02 $\left.{ }^{\circ}\right)$, C2-N1-C14 (108.76 ${ }^{\circ}$ ), and N1C14-C15 $\left(116.09^{\circ}\right)$.
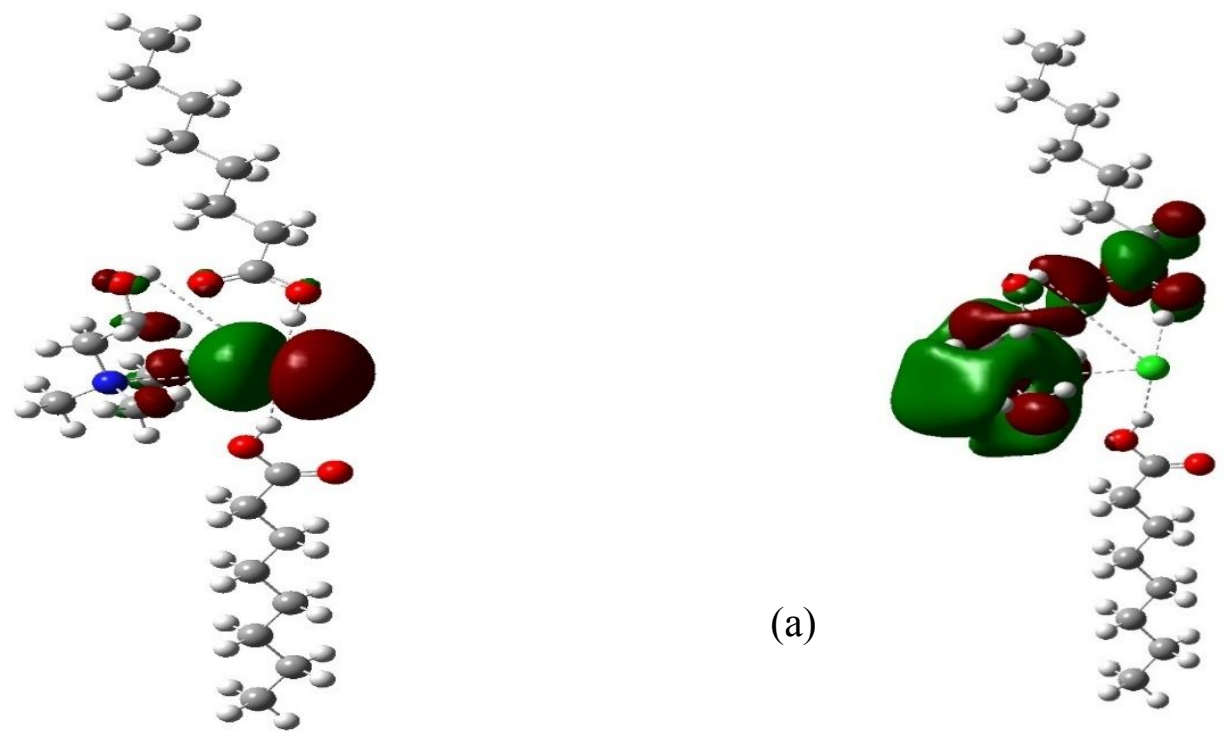

Fig. S10. Frontier molecular orbitals of [Ch]Cl:CA DES (1:2) (a) HOMO and (b) LUMO 


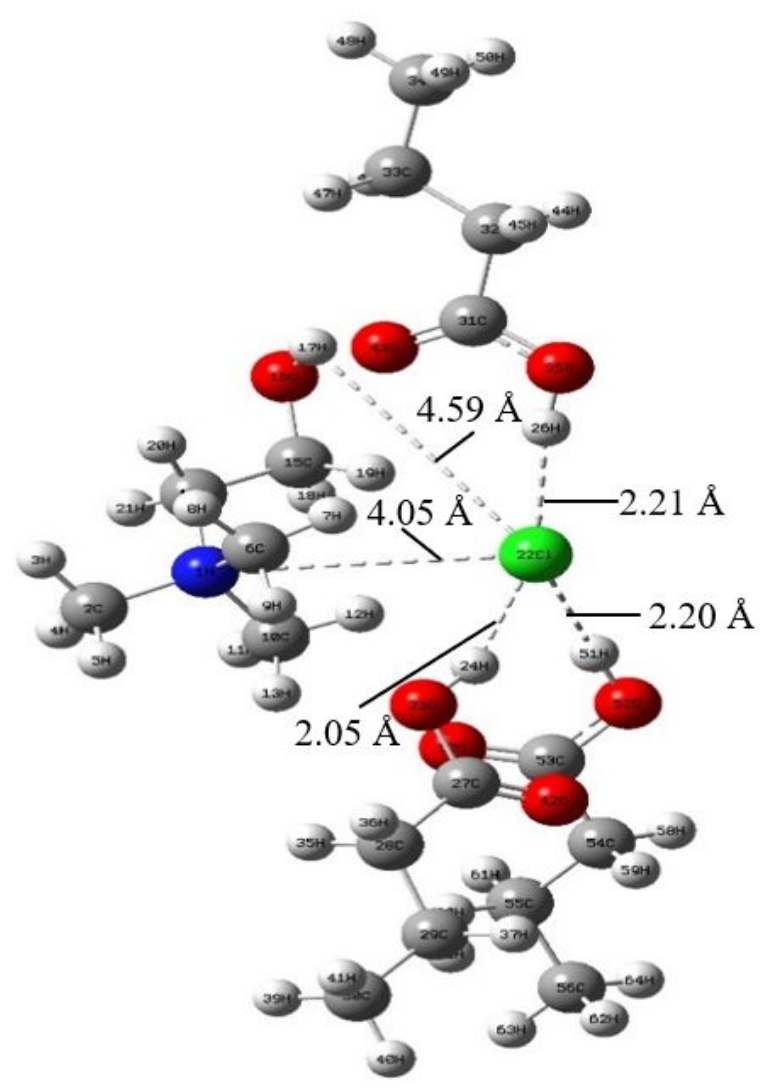

Fig. S11. Optimized equilibrium geometry structure of [Ch]Cl:BA (1:3) DES. Atoms are colored according to type: $\mathrm{C}$ (gray), $\mathrm{O}$ (red), $\mathrm{N}$ (blue), $\mathrm{H}$ (white), and $\mathrm{Cl}$ (light green). Bond angle between C14-C15-O16 (107.96 $\left.{ }^{\circ}\right)$, C15-O16-H17 (105.16 $\left.{ }^{\circ}\right), \mathrm{C} 2-\mathrm{N} 1-\mathrm{C} 14$ (108.79 ${ }^{\circ}$ ), and N1C14-C15 (116.01 $\left.{ }^{\circ}\right)$.
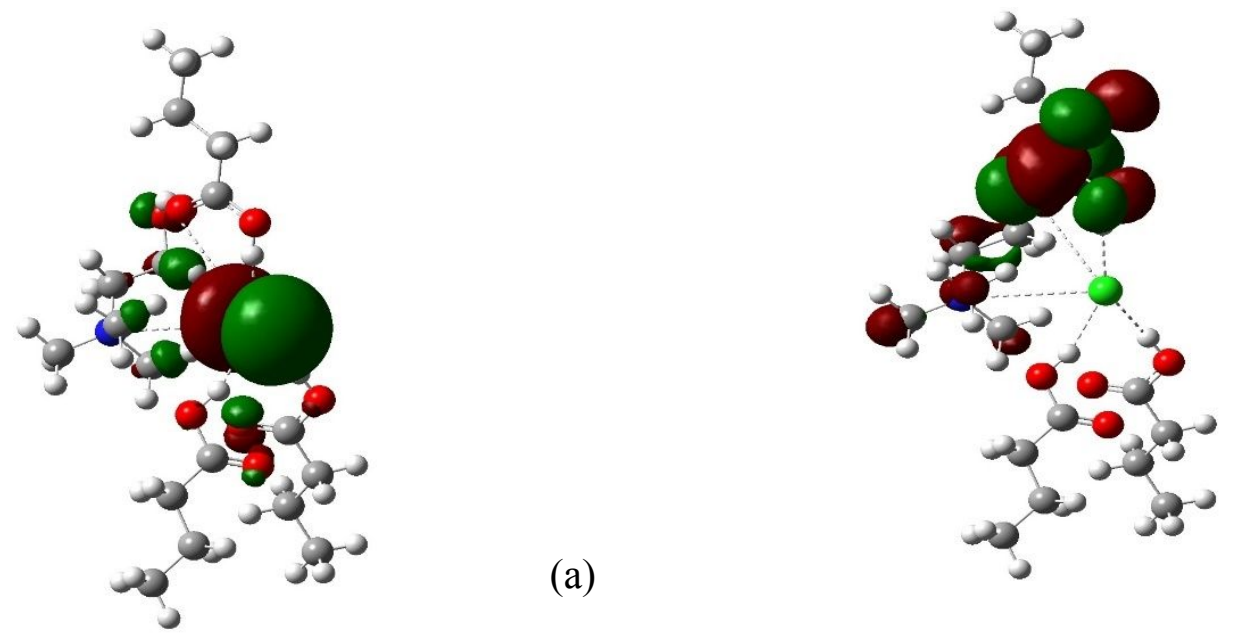

(b)

Fig. S12. Frontier molecular orbitals of [Ch]Cl:BA (1:3) DES: (a) HOMO and (b) LUMO 


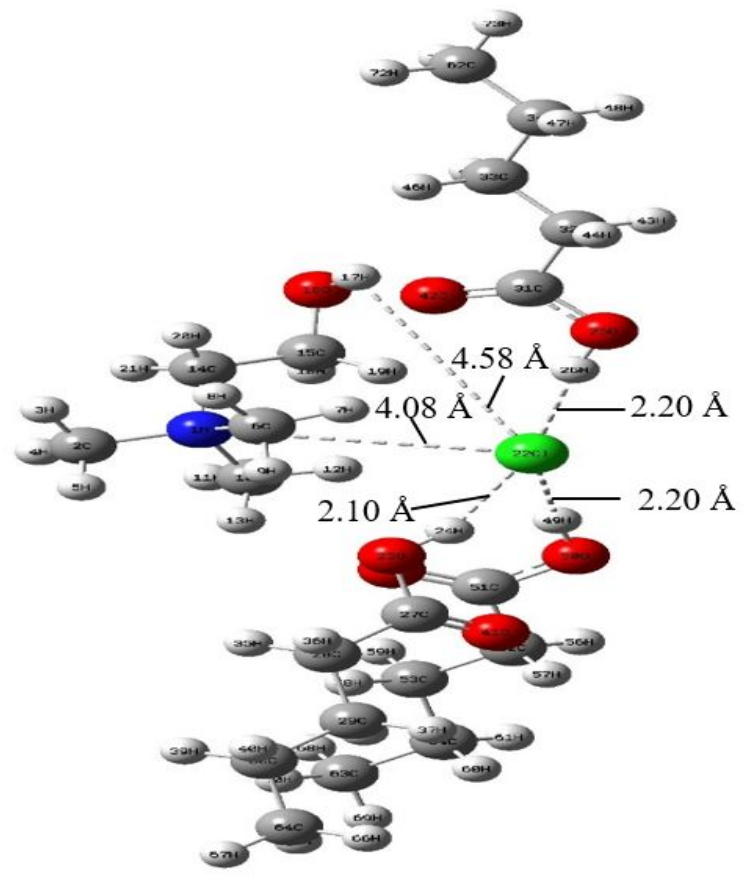

Fig. S13. Optimized equilibrium geometry structure of $[\mathrm{Ch}] \mathrm{Cl}: \mathrm{BA}$ (1:3) DES. Atoms are colored according to type: $\mathrm{C}$ (gray), $\mathrm{O}$ (red), $\mathrm{N}$ (blue), $\mathrm{H}$ (white), and $\mathrm{Cl}$ (light green). Bond angle between C14-C15-O16 (108.04 ${ }^{\circ}$ ), C15-O16-H17 (105.19 $\left.{ }^{\circ}\right), \mathrm{C} 2-\mathrm{N} 1-\mathrm{C} 14$ (108.83 ${ }^{\circ}$ ), and N1-C14-C15 $\left(115.97^{\circ}\right)$.

(a)

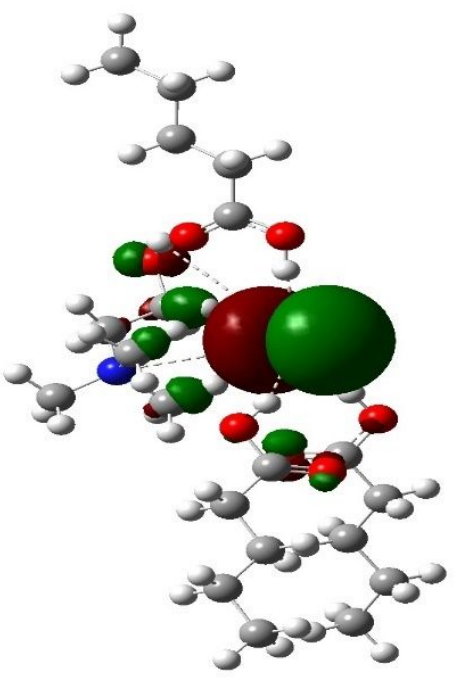

(b)

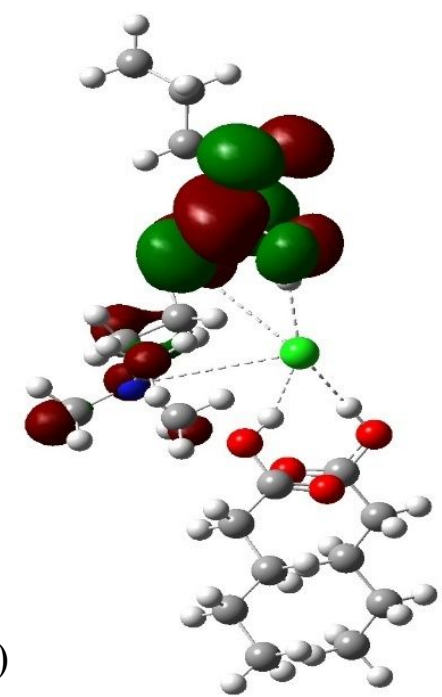

Fig. S14. Frontier molecular orbitals of 
Table S1. Viscosity of DESs synthesized in this study

\begin{tabular}{lll}
\hline DES & Mole ratio & Viscosity $(\mathrm{cP})$ \\
\hline$[\mathrm{Ch}] \mathrm{Cl}: \mathrm{BA}$ & $1: 2$ & $1757 \pm 14$ \\
& $1: 3$ & $54.1 \pm 3.5$ \\
{$[\mathrm{Ch}] \mathrm{Cl}: \mathrm{VA}$} & $1: 2$ & $1497 \pm 41$ \\
& $1: 3$ & $44.5 \pm 11$ \\
{$[\mathrm{Ch}] \mathrm{Cl}: \mathrm{CA}$} & $1: 2$ & $1594.7 \pm 31$ \\
& $1: 3$ & $1561.3 \pm 70$ \\
\hline
\end{tabular}

Table S2. Molecular parameters of choline chloride and carboxylic acids. The global hardness, $\eta$ $=\mathrm{I}-\mathrm{A} / 2$; and chemical softness, $\mathrm{S}=1 / 2 \eta$; where $\mathrm{I}=$ ionization energy and $\mathrm{A}=$ electron affinity.

\begin{tabular}{ccccccccccc}
\hline $\begin{array}{c}\text { HBA } \\
\text { and } \\
\text { HBDs }\end{array}$ & $\begin{array}{c}\epsilon \text { HOMO } \\
(\mathrm{eV})\end{array}$ & $\begin{array}{c}\epsilon \\
\mathrm{LUMO} \\
(\mathrm{eV})\end{array}$ & $\begin{array}{c}\Delta \epsilon \\
(\mathrm{eV})\end{array}$ & $\mathrm{I}$ & $\mathrm{A}$ & $\mu$ & $\chi$ & $\eta$ & $\mathrm{S}$ & $\begin{array}{c}\text { Dipole } \\
\text { moment } \\
\text { (Debye) }\end{array}$ \\
\hline$[\mathrm{Ch}] \mathrm{Cl}$ & -0.17186 & 0.01291 & 0.18477 & 0.17186 & -0.01291 & -0.07948 & 0.079475 & 0.092385 & 5.412134 & 11.5653 \\
BA & -0.27479 & 0.01038 & 0.28517 & 0.27479 & -0.01038 & -0.13221 & 0.132205 & 0.142585 & 3.50668 & 1.3672 \\
2BA & -0.28011 & 0.00365 & 0.28376 & 0.28011 & -0.00365 & -0.13823 & 0.13823 & 0.14188 & 3.524105 & 0.0002 \\
3BA & -0.26809 & 0.00071 & 0.2688 & 0.26809 & -0.00071 & -0.13369 & 0.13369 & 0.1344 & 3.720238 & 2.294 \\
VA & -0.27462 & 0.01054 & 0.28516 & 0.27462 & -0.01054 & -0.13204 & 0.13204 & 0.14258 & 3.506803 & 1.3438 \\
2VA & -0.27952 & 0.004 & 0.28352 & 0.27952 & -0.004 & -0.13776 & 0.13776 & 0.14176 & 3.527088 & 0.0245 \\
3VA & -0.26775 & 0.00125 & 0.269 & 0.26775 & -0.00125 & -0.13325 & 0.13325 & 0.1345 & 3.717472 & 2.3368 \\
CA & -0.27448 & 0.01079 & 0.28527 & 0.27448 & -0.01079 & -0.13185 & 0.131845 & 0.142635 & 3.505451 & 1.3059 \\
$2 \mathrm{CA}$ & -0.27861 & 0.00435 & 0.28296 & 0.27861 & -0.00435 & -0.13713 & 0.13713 & 0.14148 & 3.534068 & 0.0606 \\
\hline
\end{tabular}


Table S3. Molecular indices of DESs calculated from DFT data.

\begin{tabular}{cccccc}
\hline DES & $\begin{array}{c}\text { Molar } \\
\text { ratio }\end{array}$ & $\mathrm{S}$ & $\eta$ & $\begin{array}{c}\mu= \\
(\mathrm{CHOMO}+\mathrm{C} \\
\mathrm{LUMO}) / 2\end{array}$ & $\begin{array}{c}\chi \\
=(\mathrm{I}+\mathrm{A}) / 2\end{array}$ \\
\hline$[\mathrm{Ch}] \mathrm{Cl}: \mathrm{BA}$ & $1: 1$ & 4.577078 & 0.10924 & -0.10005 & 0.10005 \\
& $1: 2$ & 4.321895 & 0.11569 & -0.10416 & 0.10416 \\
& $1: 3$ & 4.039098 & 0.12379 & -0.11343 & 0.11343 \\
{$[\mathrm{Ch}] \mathrm{Cl}: \mathrm{VA}$} & $1: 1$ & 4.577497 & 0.10923 & -0.10014 & 0.10014 \\
& $1: 2$ & 4.254595 & 0.11752 & -0.10473 & 0.10473 \\
& $1: 3$ & 4.03812 & 0.12382 & -0.11338 & 0.11338 \\
{$[\mathrm{Ch}] \mathrm{Cl}: \mathrm{CA}$} & $1: 1$ & 4.548763 & 0.10992 & -0.09863 & 0.09863 \\
& $1: 2$ & 4.264392 & 0.11725 & -0.10437 & 0.10437 \\
\hline
\end{tabular}

aStandard uncertainties, $\mathrm{u}$, of $\mathrm{S}, \eta, \mu$, and $\chi$ are $\mathrm{u}(\mathrm{S})=0.016, \mathrm{u}(\eta)=0.00099, \mathrm{u}(\mu)=0.0069$, and $\mathrm{u}(\chi)=0.0067$. 
Table S4. Literature data of ionic liquids and organic solvents used for ternary plot

\begin{tabular}{|c|c|c|c|c|}
\hline Solvent & $E_{T}{ }^{N}$ & $\alpha$ & $\boldsymbol{\beta}$ & $\pi^{*}$ \\
\hline $\operatorname{bmim}]\left[\mathrm{SbF}_{6}\right]^{1}$ & 0.673 & 0.639 & 0.146 & 1.039 \\
\hline$[\mathrm{bmim}]\left[\mathrm{BF}_{4}{ }^{1}\right.$ & 0.67 & 0.627 & 0.376 & 1.047 \\
\hline$[\mathrm{bmim}]\left[\mathrm{PF}_{6}{ }^{1}\right.$ & 0.669 & 0.634 & 0.207 & 1.032 \\
\hline$[\mathrm{bmim}]\left[\mathrm{TfO}^{1}\right.$ & 0.656 & 0.625 & 0.464 & 1.006 \\
\hline$[\mathrm{bmim}]\left[\mathrm{N}(\mathrm{Tf})_{2}\right]^{1}$ & 0.644 & 0.617 & 0.243 & 0.984 \\
\hline$[\mathrm{bm} 2 \mathrm{im}]\left[\mathrm{BF}_{4}\right]^{1}$ & 0.576 & 0.402 & 0.363 & 1.083 \\
\hline$[$ bmpy $]\left[\mathrm{N}(\mathrm{Tf})_{2}\right]^{1}$ & 0.544 & 0.427 & 0.252 & 0.954 \\
\hline$[\mathrm{bm} 2 \mathrm{im}]\left[\mathrm{N}(\mathrm{Tf})_{2}{ }^{1}\right.$ & 0.541 & 0.381 & 0.239 & 1.01 \\
\hline$[\mathrm{EMIm}]\left[\mathrm{PF}_{6}\right]^{1}$ & 0.676 & 0.66 & 0.2 & 0.99 \\
\hline$[\mathrm{EMIm}]\left[\mathrm{NTf}_{2}\right]^{2}$ & 0.657 & 0.76 & 0.28 & 0.9 \\
\hline$[\mathrm{EMIm}]\left[\mathrm{ClO}_{4}\right]^{2}$ & 0.67 & 0.56 & 0.41 & 1.11 \\
\hline$[\mathrm{EMIm}][\mathrm{DCA}]^{2}$ & 0.648 & 0.53 & 0.35 & 1.08 \\
\hline$[\mathrm{EMIm}]\left[\mathrm{NO}_{3}\right]^{2}$ & 0.642 & 0.48 & 0.66 & 1.13 \\
\hline$[\mathrm{EMIm}][\mathrm{AC}]^{2}$ & 0.59 & 0.4 & 0.95 & 1.09 \\
\hline$[\mathrm{HOEMIm}]\left[\mathrm{PF}_{6}\right]^{2}$ & 0.957 & 1.17 & 0.15 & 1.11 \\
\hline$[\mathrm{HOEMIm}]\left[\mathrm{NTf}_{2}\right]^{2}$ & 0.929 & 1.17 & 0.34 & 1.03 \\
\hline$[\mathrm{HOEMIm}]\left[\mathrm{ClO}_{4}\right]^{2}$ & 0.914 & 1.06 & 0.16 & 1.13 \\
\hline$\left[\right.$ HOEMIm] $[\text { DCA }]^{2}$ & 0.784 & 0.8 & 0.51 & 1.11 \\
\hline$[\mathrm{HOEMIm}]\left[\mathrm{NO}_{3}\right]^{2}$ & 0.769 & 0.77 & 0.65 & 1.11 \\
\hline 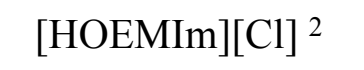 & 0.769 & 0.73 & 0.68 & 1.16 \\
\hline$\left[\right.$ HOEMIm][AC ${ }^{2}$ & 0.633 & 0.53 & 0.9 & 1.04 \\
\hline
\end{tabular}


Table S4. Continued:

\begin{tabular}{|c|c|c|c|c|}
\hline Solvent & $\overline{E_{T} \mathrm{~N}}$ & $\alpha$ & $\bar{\beta}$ & $\pi^{*}$ \\
\hline$[3-\mathrm{MBP}]\left[\mathrm{BF}_{4}\right]^{3}$ & 0.651 & 0.56 & 0.423 & 1.071 \\
\hline$[4-\mathrm{MP}]\left[\mathrm{BF}_{4}\right]^{3}$ & 0.636 & 0.53 & 0.533 & 1.066 \\
\hline Acetonitrile ${ }^{1}$ & 0.46 & 0.35 & 0.37 & 0.799 \\
\hline Acetone ${ }^{1}$ & 0.35 & 0.202 & 0.539 & 0.704 \\
\hline Dichloromethane $^{3}$ & 0.309 & 0.04 & 0.578 & 0.733 \\
\hline Toluene ${ }^{1}$ & 0.1 & -0.213 & 0.077 & 0.532 \\
\hline Methanol ${ }^{3}$ & 0.762 & 1.03 & 0.578 & 0.771 \\
\hline $\begin{array}{c}1,1,2,2- \\
\text { Tetrachloroethane }^{4}\end{array}$ & 0.309 & 0 & 0 & 0.95 \\
\hline Nitrobenzene ${ }^{4}$ & 0.324 & 0 & 0.3 & 1.01 \\
\hline $\begin{array}{c}1,1,1- \\
\text { Trichloroethane }\end{array}$ & 0.17 & 0 & 0 & 0.49 \\
\hline Benzene ${ }^{4}$ & 0.111 & 0 & 0.1 & 0.59 \\
\hline Acetophenone ${ }^{4}$ & 0.306 & 0.04 & 0.49 & 0.9 \\
\hline Toluene ${ }^{4}$ & 0.0099 & 0 & 0.11 & 0.54 \\
\hline 1,4-dioxane ${ }^{4}$ & 0.164 & 0 & 0.37 & 0.55 \\
\hline Cyclohexanone ${ }^{4}$ & 0.281 & 0 & 0.53 & 0.76 \\
\hline $\mathrm{DMSO}^{4}$ & 0.444 & 0 & 0.76 & 1 \\
\hline Ethyl acetate ${ }^{4}$ & 0.228 & 0 & 0.45 & 0.55 \\
\hline Tetrahydrofuran ${ }^{4}$ & 0.207 & 0 & 0.55 & 0.58 \\
\hline Ethanol 5 & - & 0.98 & 0.83 & 0.51 \\
\hline
\end{tabular}




\section{References}

(1) Crowhurst, L.; Mawdsley, P. R.; Perez-Arlandis, J. M.; Salter, P. A.; Welton, T., Solventsolute interactions in ionic liquids. Phys. Chem. Chem. Phys. 2003, 5, 2790-2794.

(2) Zhang, S.; Qi, X.; Ma, X.; Lu, L.; Deng, Y., Hydroxyl ionic liquids: the differentiating effect of hydroxyl on polarity due to ionic hydrogen bonds between hydroxyl and anions. $J$. Phys. Chem. B 2010, 114, 3912-3920.

(3) Khupse, N. D.; Kumar, A., Delineating solute- solvent interactions in binary mixtures of ionic liquids in molecular solvents and preferential solvation approach. J. Phys. Chem. B 2010, $115,711-718$.

(4) Chiappe, C.; Pieraccini, D., Determination of Ionic Liquids Solvent Properties Using an Unusual Probe: The Electron Donor-Acceptor Complex between 4, 4 '-bis (Dimethylamino)benzophenone and Tetracyanoethene. J. Phys. Chem. A 2006, 110, 4937-4941.

(5) Buhvestov, U.; Rived, F.; Ràfols, C.; Bosch, E.; Rosés, M., Solute--solvent and solventsolvent interactions in binary solvent mixtures. Part 7. Comparison of the enhancement of the water structure in alcohol-water mixtures measured by solvatochromic indicators. J. Phys. Org. Chem. 1998, 11, 185-192. 